\title{
Multimaterial 3D Printed Soft Actuators Powered by Shape Memory Alloy Wires
}

\author{
Saeed Akbari ${ }^{1 * \&}$, Amir Hosein Sakhaei2,3\&, Sahil Panjwani2\&, \\ Kavin Kowsari², Ahmad Serjourei ${ }^{2}$, Qi Ge ${ }^{2,4 * *}$
}

1. RISE Research Institutes of Sweden, Box 104, SE-431 22 Mölndal, Sweden

2. Digital Manufacturing and Design Center, Singapore University of Technology and Design, Singapore 487372, Singapore

3. College of Engineering, Mathematics and Physical Sciences, University of Exeter, Exeter, EX4 4QF, UK

4. Science and Math Cluster, Singapore University of Technology and Design, Singapore 487372, Singapore

\footnotetext{
*E-mail: $\underline{\text { saeed.akbari@ri.se }}$

**E-mail: ge qi@sutd.edu.sg

\&These authors contributed equally to this work.
}

\begin{abstract}
Shape memory alloys (SMAs) have been widely used to fabricate soft actuators by embedding SMA wires into various soft matrices manufactured by conventional moulding methods or novel threedimensional (3D) printing techniques. However, soft matrices of SMA based actuators are typically fabricated from only one or two different materials. Here, we exploit the great manufacturing flexibility of multimaterial 3D printing to fabricate various bending, twisting and extensional actuators by precisely controlling the spatial arrangements of different printing materials with different stiffnesses. In order to achieve a broad range of deformations, ten different printing materials were characterized and used in the actuators design. In addition, we developed a finite element model to simulate complex deformations of the printed actuators and facilitate the design process. The model incorporates a user defined material subroutine that describes the nonlinear temperature dependant behavior of SMAs. The results show the efficiency and flexibility of multimaterial 3D printing in tailoring the deformed shape of the SMA based soft actuators, which cannot be accomplished using conventional manufacturing methods such as moulding.
\end{abstract}

Keywords: 3D printing, shape memory alloy, soft actuator, finite element modeling. 


\section{Introduction}

Shape memory alloys (SMAs) are a class of active materials that can perform reversible actuation upon thermomechanical training [1]. Although SMAs are manufactured in a variety of shapes, including plates, springs, wires, and ribbons, the most widely used shape is the onedimensional SMA wire, which can be integrated into soft matrices to fabricate actuators that transform one-dimensional deformation of the SMA wires into a broad range of three-dimensional deformations. For instance, Rodrigue et al. developed soft actuators comprised of a polydimethylsiloxane (PDMS) matrix embedded with Nitinol (Nickel-Titanium) SMA wires arranged in different configurations to create a variety of bending [2-5], twisting [6-8], and extensional [9] actuators, which have potential applications in soft robotics [10-12], aerospace [13, 14], and biomedical $[15,16]$ fields.

Most research efforts to develop SMA powered soft actuators rely on the time-consuming moulding and casting based manufacturing methods $[2-9,17,18]$, which typically consist of the following steps: (i) fabricating a polymeric or metallic mold based on the desired geometry of soft actuator; (ii) embedding a pre-strained SMA wire into the mold and fixing the two ends of the SMA wire on both ends of the mold; (iii) casting liquid polymer resin into the mold, and thermally curing the resin; and (iv) finally removing the actuator from the mold. Major limitation of this method is that each actuator design requires fabricating a different mold adopting the actuator geometry. Moreover, this method can produce only simple structures.

Recent advancements in multimaterial three-dimensional (3D) printing technologies have significantly improved our capability to fabricate complicated 3D structures with intricate details through micron-scale placement of various materials with different properties. In particular, multimaterial inkjet 3D printing provides high design freedom to produce complex composite structures by selectively depositing photopolymer droplets on a build tray followed by UV illumination to trigger photopolymerization, which transforms the droplets of various constituents and compositions into solids with different mechanical properties [19-26]. Using this technology, the spatial arrangement of different materials over multiple length scales can be easily achieved in a 
single printing job, and the desired stiffness variation can be realized [27-29]. Multimaterial inkjet 3D printing has the potential to automate the fabrication process of the body of the soft actuators, and replace the conventional manufacturing methods.

Although some recent studies have used 3D printing in fabricating SMA based actuators [3033], they have not tried to tailor the actuator deformation using different printing materials. Also, developing a predictive model to simulate the actuator deformation and study the effect of printing materials properties on its performance is of critical importance. A comprehensive simulation study to capture the actuator tailored performance and facilitate the design process is lacking in the literature.

In this paper, we present a design and manufacturing approach to fabricate SMA powered soft actuators with tunable functionalities and various deformations. We take advantage of this technology by following two different strategies to create various deformation modes including bending, twisting and extensional deformations. First, we modulate the stiffness of the soft and rigid segments of the multimaterial actuators by printing various compositions of the two base materials having different properties. Second, we control the spatial variation of the soft and rigid segments to achieve the desired structural performance. We start with three basic designs of the multimaterial soft actuators for bending, twisting and extensional deformations, then show the experimental results of the effect of different design parameters, including material properties and geometry, on the performance of the printed actuators. As an example of the versatility of multimaterial 3D printing, we fabricate a soft gripper capable of effective grasping performance. We also develop a finite element model that incorporates a user defined (UMAT) subroutine to accurately capture nonlinear temperature dependant behaviour of SMA wires to simulate complex deformations of the printed actuators. The proposed guidelines provide a foundation for effective design and performance of SMA powered 3Dprinted soft actuators.

\section{Materials and Methods}

\subsection{Design of Soft Actuators}


A major advantage of multimaterial 3D printing to fabricate soft actuators is its ability to precisely control the spatial arrangements of distinctive soft and rigid segments without complicating the manufacturing process or increasing the fabrication time. This cannot be easily achieved in conventional fabrication methods. For example, in order to optimize the bending actuation of the SMA-embedded soft actuators, a rigid thin layer is commonly placed in the mid-plane of the actuator to increase the axial stiffness, and reduce the axial contraction caused by the contraction of the SMA wire [3]. Moreover, both ends of the actuator must be fabricated from a rigid material to ensure that during the actuation process, the metal connections fixing the SMA wire to the actuator body do not damage the soft matrix at elevated temperatures $[3,5,18]$. These requirements add steps to the conventional manufacturing processes, therefore increase the time and cost of fabrication. These challenges can be easily addressed using the multimaterial inkjet 3D printing technology which provides a promising solution to simplify the complexity of fabricating soft actuators by directly $3 \mathrm{D}$ printing multimaterial structures where the materials with different stiffnesses are precisely placed at desired locations.

Fig. 1 depicts the basic designs of the multimaterial soft actuators used in this study to achieve bending and extensional deformations. Each actuator consists of a multimaterial structure, including a polymeric soft matrix and several rigid reinforcement segments, which are fabricated using a multimaterial inkjet 3D printer. After the actuator is printed, the SMA wire is then inserted into the printed polymeric structure, and fixed externally at both ends using crimp connections to prevent sliding between the embedded wire and the printed structure. During actuation, the small linear axial deformation of the SMA wire is transformed into a large out-of-plane deformation of the actuator.

By changing the location of the SMA wire within the actuator as well as the geometry and spatial arrangement of the rigid reinforcing segments, we can create actuators exhibiting different complex 3D deformations. As shown in Fig. 1a, in order to create a large-deformation bending actuator, the SMA wire is eccentrically embedded into the printed structure where a thin layer of the rigid material is printed along the midplane of the actuator. Fig. $1 \mathrm{~b}$ presents another method to create an actuator that transforms the axial deformation of SMA wire into bending deformation through concentrically inserting an SMA wire into the printed structure where the rigid thin layers are placed 
alternatively on the top and bottom surfaces. Incorporating a number of these actuators as the repeating unit into a larger structure, we can fabricate actuators exhibiting net extensional deformation. The details are explained in Section 2.3. Additionally, in Section 2.3, we demonstrate the concept of twisting actuators created by printing the reinforcing rigid fibers at a certain angle from the actuator longitudinal direction.

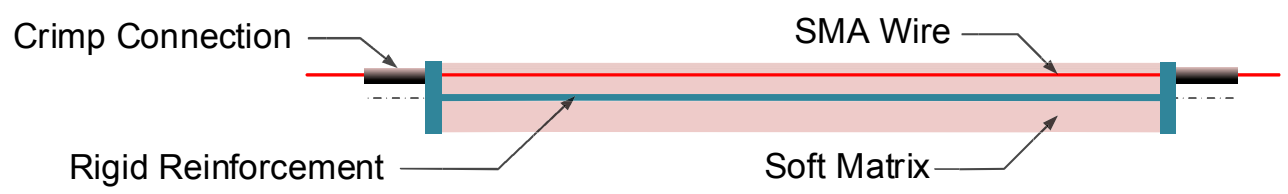

(a)

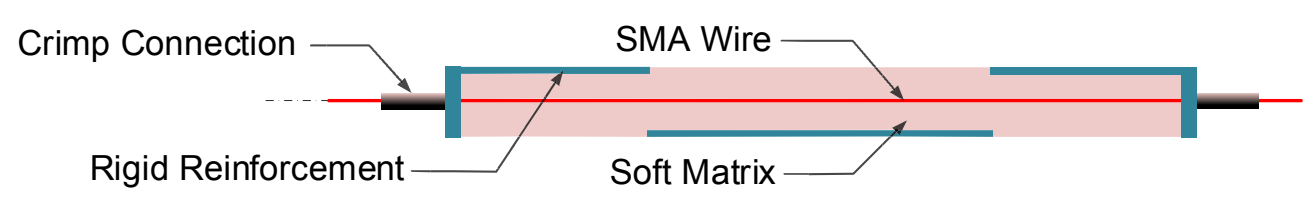

(b)

Fig. 1. Basic designs of the SMA-based 3D printed soft multimaterial actuators incorporating rigid reinforcement segments into a soft matrix. a) Bending actuator with an eccentrically embedded SMA wire and a rigid layer printed at the midplane. b) Extensional actuator with a concentrically embedded SMA wire and alternating rigid reinforcement segments printed on the top and bottom surfaces.

\subsection{Characterization of 3D Printing Materials}

Mechanical properties of the printed materials are required in the design process of the actuators to achieve a desired performance, as well as in the finite element model to simulate deformation of the actuators. The tensile behaviour of the 3D printed materials was characterized according to ASTM D638-10 test standard for tensile testing of plastics. Three samples of each material were printed using the multimatrial inkjet printer (Objet500 Connex 3, Stratasys, Edina, MN, USA), and tested with a universal testing machine (Criterion Model 43, MTS Systems Corporation, Eden Prairie, MN, USA) at a loading speed of $2 \mathrm{~mm} \cdot \mathrm{min}^{-1}$.

The printing materials available on the printer include a rubber-like elastomer, referred to as Agilus30, and a rigid polymer, referred to as VeroClear, as well as a number of digital materials 
which are mixtures of VeroClear and Agilus30. Soft printed materials include Agilus30 and digital materials with Agilus30 as the base material (identified with prefix FLX). Rigid printed materials include VeroClear and digital materials with VeroClear as the base material (identified with prefix RGD). Figs. 2a-b and 2c-d show the results of tensile tests for the soft and rigid materials, respectively, including stress-strain behaviour and Young's modulus. It is observed that the Young's modulus of VeroClear $(E=1.4 \mathrm{GPa})$ is about four orders of magnitudes larger than Agilus30 $(E=0.43$ $\mathrm{MPa})$. The moduli of the digital materials are between $(0.43 \mathrm{MPa}<E<1.4 \mathrm{GPa})$. Since Young's modulus of the rigid materials is significantly larger than the soft materials, the stiffness of the printed structures described in Fig. 1 can be controlled by adjusting the thickness of the rigid reinforcements segments.

(a)

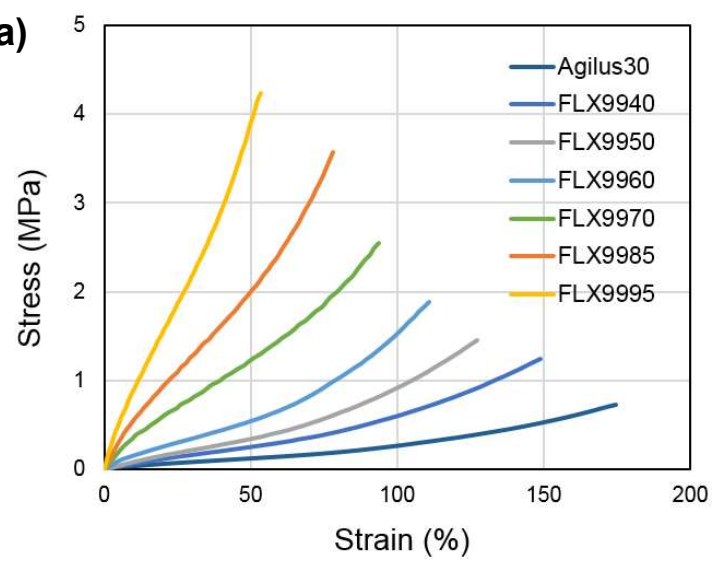

(c)

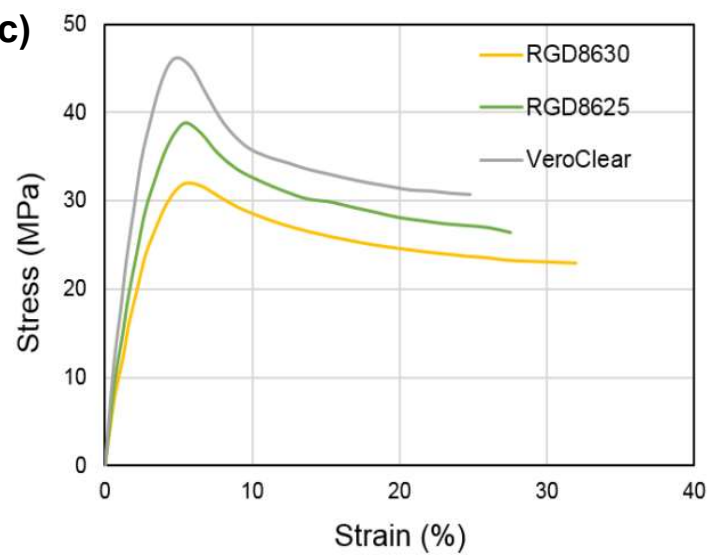

(b)

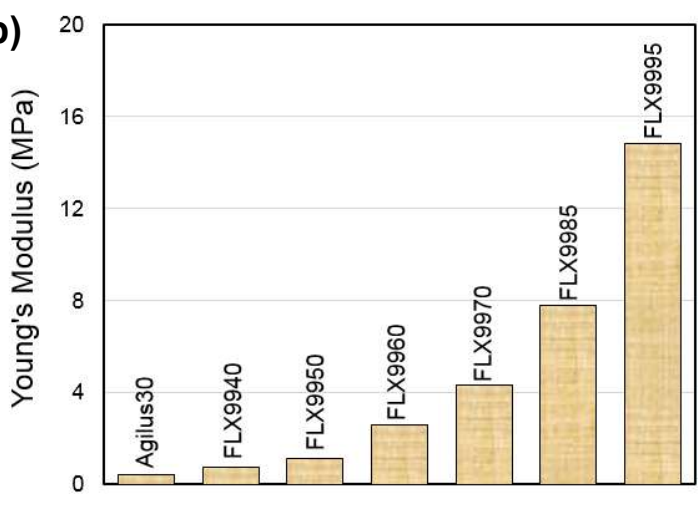

(d)

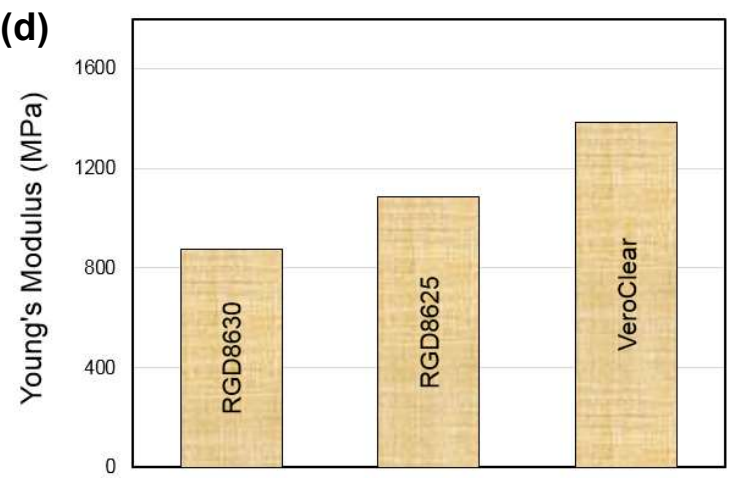

Fig. 2. Results of the characterization test of the printing materials. a) Stress-strain curves of the soft materials used to print the soft matrix of the actuators. b) Young's modulus of the soft materials. c) Stress-strain curves of the rigid materials used to print the rigid reinforcement segments of the actuators. d) Young's modulus of the rigid materials. 


\subsection{Fabrication of Soft Actuators}

The fabrication of a soft actuator starts by printing multimaterial structures containing soft and rigid segments. Then, the SMA wire is inserted into the printed multimaterial body of the actuator. The SMA wire used in this study is a Nickel-Titanium alloy (Flexinol, Irvine, CA, USA) with a diameter of $0.25 \mathrm{~mm}$ which functions as tendon actuator to generate bending, twisting, or extensional deformation based on the spatial layout of the SMA wire and the rigid reinforcement segments within the soft matrix, as shown in Fig. 1.

In each multimaterial structure a $0.4 \mathrm{~mm}$ diameter hole along the length of the actuator is designed for positioning the SMA wire. The SMA wire is first pre-strained by $5 \%$. Then, depending on the type of the actuator (Fig. 1), it is embedded eccentrically, with a fixed off-centre distance of 0.7 $\mathrm{mm}$, or concentrically into the printed structure. The embedding process is done manually. After inserting the SMA wire, it is fixed at both ends using copper crimp connections (Insulated Crimp Bootlace Ferrule, RS Components, Corby, UK) which had a pin diameter and length of $1.1 \mathrm{~mm}$ and 8 $\mathrm{mm}$, respectively. The crimping was performed using a standard crimping tool (PZ6/5 Ratchet, Weidmüller, Detmold, Germany) to ensure there is no slack between the embedded SMA wire and the actuator body.

Applying an electric current of 1.0 A to the pre-strained SMA wire embedded within the actuator raises its temperature via Joule heating until it exceeds its austenite transition temperature. Consequently, the SMA wire contracts to recover its original undeformed shape. Since the wire is fixed to the printed multimaterial structure at both ends using crimp connections, its contraction is transferred to the multimaterial structure and produces a large deformation. After the electric current is switched off, the SMA wire cools down below the austenite transition temperature, and the elastic strain energy stored in the deformed actuator results in the recovery of its original shape.

Fig. 3 presents the details the bending actuators following the basic design introduced in Fig. 1a. The first design shown in Fig. 3a has a uniform cross section over its effective length, with a thin rigid layer printed from VeroClear, and two soft layers with equal thicknesses symmetrically printed on the top and bottom of the rigid layer, as indicated in cross-section A-A. Also, a $2 \mathrm{~mm}$ long rigid 
segment at both ends of the soft matrix is printed from VeroClear to prevent direct contact between the crimp connection and the soft matrix. The SMA wire in this design is embedded into the top soft layer to create a bending deformation of the structure upon actuation. The dimensions of each actuator are $60 \mathrm{~mm}$ in length (unless otherwise mentioned), $15 \mathrm{~mm}$ in width, and $3 \mathrm{~mm}$ in thickness, with an extra $35 \mathrm{~mm}$ long rigid feature printed at the end of the actuator to facilitate a secure connection between the actuator and a rigid surface. The distance between the SMA wire and the midplane of the actuator is $0.7 \mathrm{~mm}$.

Fig. $3 \mathrm{~b}$ depicts the second design of the bending actuator having two hinges which can be printed from two different elastomeric digital materials (properties of Fig. 2a-b) to achieve dissimilar bending angles at each hinge. Similar to the first design, a thin rigid layer was printed along the midplane over the entire length of the actuator to avoid axial contraction and buckling of the soft matrix during actuation. For simplicity, the effective length of the actuator is designed to consist of five segments with equal lengths, including three rigid sections and two soft sections functioning as hinges. The layer on the top of the thin rigid layer is always printed from a soft digital material, while the layer on the bottom is printed from a rigid material to create rigid sections, or from a soft digital material to create soft hinges, as depicted in cross-sections B-B and C-C. 


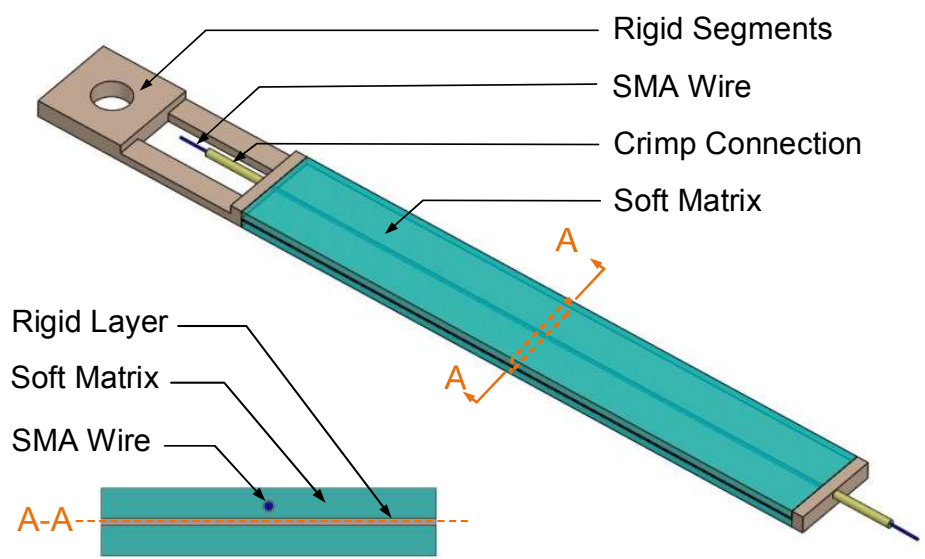

(a)

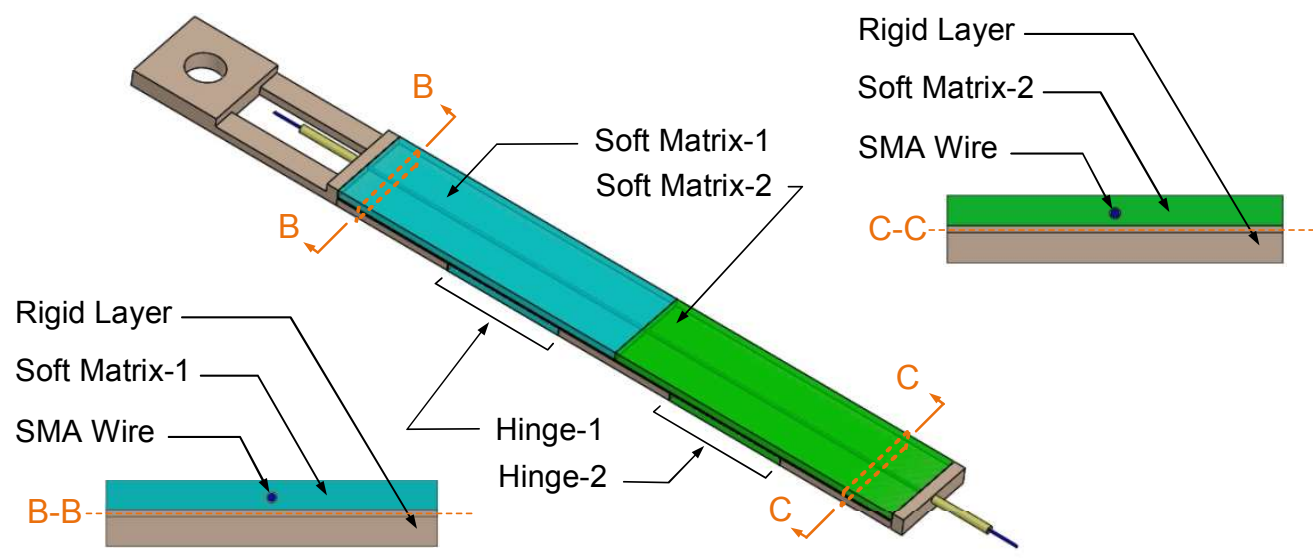

(b)

Fig. 3. Design of the SMA-based 3D printed soft multimaterial bending actuators. a) Schematic of the bending actuator with a uniform cross section over the entire effective length of the actuator. It is comprised of a rigid mid-layer and two similar soft layers symmetrically placed at the top and bottom, as shown in cross-section A-A. b) Schematic of the bending actuator having five segments with equal lengths, including two soft segments acting as hinges. The other three sections have two layers, including one rigid layer, and one soft layer, as shown in cross-sections B-B and C-C. The hinges can be printed from dissimilar elastomeric digital materials to create different deformations in each hinge.

Fig. 4 presents the detailed design of a twisting actuator realized by printing thin rigid fibers from VeroClear on the bottom surface in an angle $\theta$ with respect to the actuator longitudinal direction. The angle $\theta$ can be changed to generate a broad range of twisting deformations. It should be noted that it is very difficult to create this multimaterial structure using traditional manufacturing methods. 


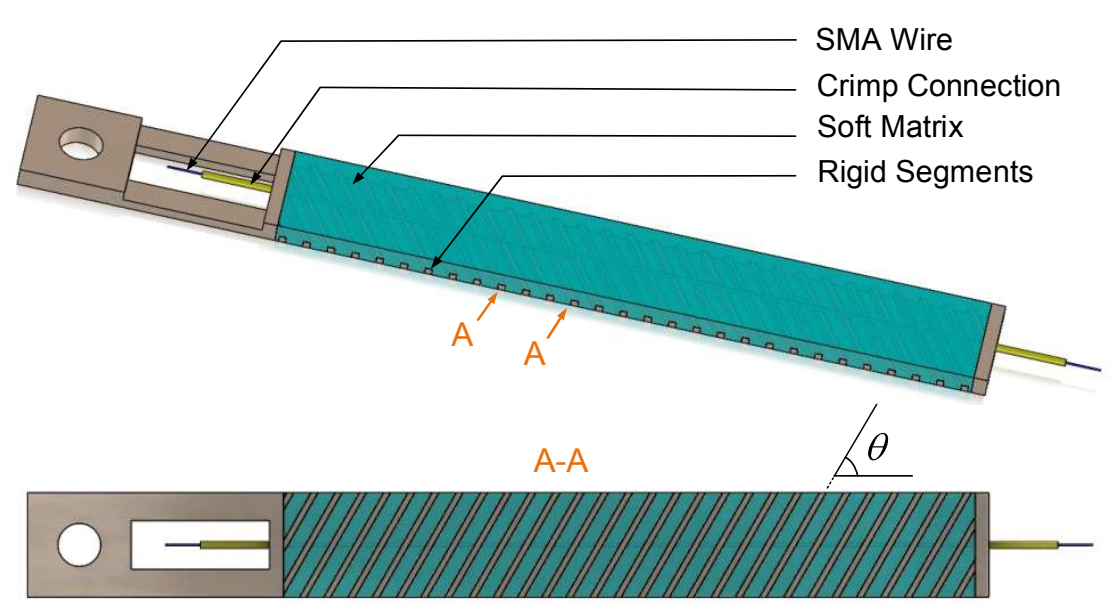

Fig. 4. Design of the SMA-based 3D printed twisting actuator, composed of a soft matrix with rigid fibers diagonally printed on its bottom surface to create a twisting deformation. The thickness of rigid fibers as well as their spacing can be modified to control twisting deformation.

Fig. 5 shows the design of the extensional actuator, which is a multi-segment structure consisting of four basic linear elements based on the design of Fig. 1b. The alternative placement of the rigid layers in each basic element results in a wavy shape of the actuation. Once actuated, it expands in transverse (vertical) direction, and contracts in longitudinal (horizontal) direction.

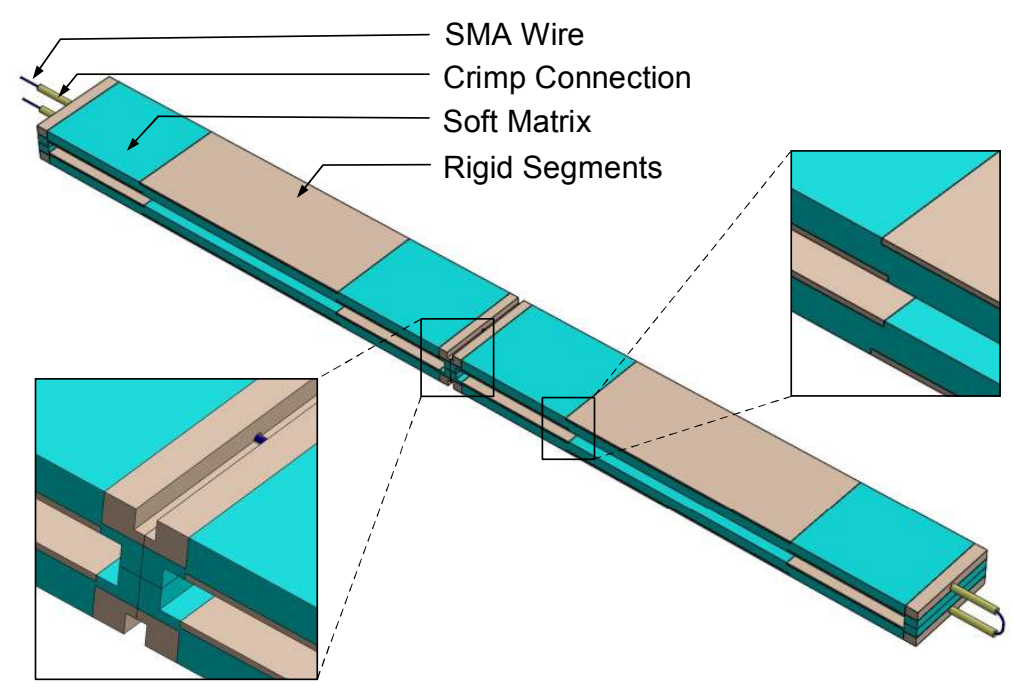

Fig. 5. Design of the SMA-based 3D printed extensional actuator, composed of four basic actuators in which thin rigid layers are printed alternatively on the top and the bottom surfaces to create a wavy shape upon actuation. A single SMA wire passes through the two layers of the actuator.

It should be noted that the proposed designs in this research for soft actuators require complicated arrangement of various materials, which are very difficult to achieve through 
conventional manufacturing methods. The superior manufacturing flexibility offered by multimaterial 3D printing to fabricate geometries with delicate details is essential to the successful implementation of the proposed designs.

\section{Finite Element Modelling}

Deformation of the bending multimaterial actuators was simulated using a two-dimensional finite element model created in the software ABAQUS (Simulia, Providence, RI, USA). The printed matrix was modeled using $1670 \mathrm{CPE} 4 \mathrm{H}$ two-dimensional plain strain elements with assigned thickness, and the SMA wire using $97 \mathrm{~B} 21 \mathrm{H}$ linear beam elements with a circular cross-section.

In the experiments, the $0.25 \mathrm{~mm}$ thick SMA wire was inserted into the actuator hole whose diameter $(0.4 \mathrm{~mm})$ was larger than the wire thickness to ensure that it can be easily embedded into the actuator. The actuator hole was initially filled with support material before the SMA wire was manually embedded into

it. Therefore, the gap between the SMA wire and the hole wall was filled with support material. However, for simplicity, in the modeling it was assumed the hole diameter and the wire thickness are both $0.25 \mathrm{~mm}$, meaning there was no gap between the SMA wire and the hole wall. A surface to surface frictionless contact was considered at the interface between the hole wall and the SMA wire. Further simulations using different values of the friction coefficient showed that the friction coefficient has a negligible effect on the actuator tip displacement. For example by increasing the friction coefficient from zero to 0.6 , the tip displacement changed by less than $0.2 \%$. This shows that for this specific actuator design, there is negligible tangential (friction) force between the SMA wire and the hole wall. Furthermore, at both ends the wire was fixed to the actuator using rigid connections. A mesh convergence study showed that using a denser mesh changed the results less than $0.1 \%$.

The soft and rigid segments of the matrix were assumed to have linear elastic behavior with the material properties reported in Fig. 2b. Furthermore, a coupled thermo-mechanical material model was used for the shape memory alloy, which was developed and implemented as a user-defined material (UMAT) subroutine according to $[34,35]$. Table 1 presents the material constants of the SMA used in the finite element model.

Table 1. Material constants of the SMA [34]. 


\begin{tabular}{|l|l|}
\hline Elastic stiffness of the austenite: $\mathrm{E}^{\mathrm{A}}$ & $75 \mathrm{GPa}$ \\
\hline Elastic stiffness of the martensite: $\mathrm{E}^{\mathrm{M}}$ & $28 \mathrm{GPa}$ \\
\hline Poisson's ratio (equal for both phases): $v$ & 0.33 \\
\hline Coefficient of thermal expansion for the austenite: $\alpha^{\mathrm{A}}$ & $2 \times 10^{-5} \mathrm{~K}^{-1}$ \\
\hline Coefficient of thermal expansion for the austenite: $\alpha^{\mathrm{M}}$ & $2 \times 10^{-5} \mathrm{~K}^{-1}$ \\
\hline Martensitic start temperature: $\mathrm{M}^{\mathrm{s}}$ & $325 \mathrm{~K}$ \\
\hline Martensitic finish temperature: $\mathrm{M}^{\mathrm{f}}$ & $315 \mathrm{~K}$ \\
\hline Austenitic start temperature: $\mathrm{A}^{\mathrm{s}}$ & $341 \mathrm{~K}$ \\
\hline Austenitic finish temperature: $\mathrm{A}^{\mathrm{f}}$ & $351 \mathrm{~K}$ \\
\hline Maximum transformation strain: $\varepsilon^{\text {tr }}$ & 0.05 \\
\hline Stress influence coefficient for austenite: $\rho \Delta \mathrm{s}^{\mathrm{A}}$ & $-350000 \mathrm{~Pa} \mathrm{~K}^{-1}$ \\
\hline Stress influence coefficient for martensite: $\rho \Delta \mathrm{s}^{\mathrm{M}}$ & $-350000 \mathrm{~Pa} \mathrm{~K}^{-1}$ \\
\hline
\end{tabular}

To simulate loading conditions, the SMA wire was first extended 5\% at room temperature, while the material was in martensitic phase. Then, the SMA wire was unloaded when it was still at room temperatures. These two steps led to detwinning in martensitic phase and induced $5 \%$ strain to the SMA wire. Finally, the contact between the SMA wire and the matrix was activated, and the SMA temperature was increased to a temperature higher than the austenitic finish temperature $\left(A_{s}=341 \mathrm{~K}\right.$, Table 1$)$. As a result, the SMA wire contracted to recover its original length. The final length of the wire depends on the bending stiffness of the matrix, which acts as a resistant force to limit the wire deformation.

\section{Results and Discussion}

Inherent low stiffness of soft actuators often results in a small actuation force, which limits their effective applications. In this research, efficient stiffness modulation of soft actuators with given general dimensions is accomplished either by changing the geometry and layout of the rigid segments within the structure, or by printing the soft matrix from various digital materials with different tensile moduli. A broad range of bending, twisting, and extensional deformations realized through accurate placement of different printing materials by multimaterial 3D printing is demonstrated in this section. 
All actuators were fabricated using the materials and manufacturing process described in Section 2.3, and were actuated through Joule heating by applying an electric current of $1.0 \mathrm{~A}$ until no further deformation was observed in the actuator. The required time for actuation was around 10-12 s. The actuators were then allowed to cool down by natural convection, and restore their initial shape.

\subsection{Bending Actuator}

We develop bending soft actuators by embedding thin SMA wires eccentrically into a polymeric soft matrix, as shown schematically in Figs. 1a and 3. A series of experiments were performed to investigate the effect of parameters such as the mid-layer thickness, the actuator length, and the matrix stiffness on the performance. Fig. 6 shows the effect of the thickness of the rigid midlayer, illustrated in Section A-A in Fig. 3a, on the actuator maximum deformation. In all three samples tested, the matrix was printed from FLX9940 with $E=0.74$ MPa (Fig. 2). Fig. 6a indicates that when there is no rigid layer, the low stiffness of the matrix causes the actuator to buckle under the axial contraction force applied by the SMA wire. However, incorporation of a thin rigid layer printed from VeroClaear with a thickness of $0.3 \mathrm{~mm}$ (Fig. 6b) increases the stiffness of the actuator and results in a larger bending deformation. Further increase of the thin layer thickness to $0.5 \mathrm{~mm}$ increases the actuator bending stiffness significantly, limits the recovery of the SMA wire (Fig. 6c), and reduces the maximum defamation of the actuator. For consistency, all the following bending actuators were fabricated with a $0.3 \mathrm{~mm}$ thick rigid mid-layer.

Fig. 7 illustrates the experimental and numerical results of the actuator length effect on the maximum deformation (Movie $\mathrm{S} 1$ shows the deformation of the $80 \mathrm{~mm}$ long actuator). As expected, longer actuators show a larger tip deformation. The good agreement between the experimental and simulation results show the efficiency of the developed finite element model in predicting the actuator performance. The verified model can be used to design printed actuators with more complex deformations. It can also be employed to estimate the force and energy generated by the actuator. Further simulations are ongoing to evaluate these parameters and compare them with the data obtained from characterization tests usually performed for this type of actuators $[3,4,36,37]$. 
The multimaterial printing method used in this work enables the fabrication of the soft matrix from various digital materials with properties reported in Fig. 2. The experimental and numerical results of the deformed shape of the actuators with soft matrix printed from different digital materials is shown in Fig. 8. The actuators with soft matrix printed from more flexible digital materials show a larger deformation, although at the sacrifice of stiffness, as shown quantitatively in Fig. 8d. This enables tunable functionality of the actuator based on the structural and performance requirements.
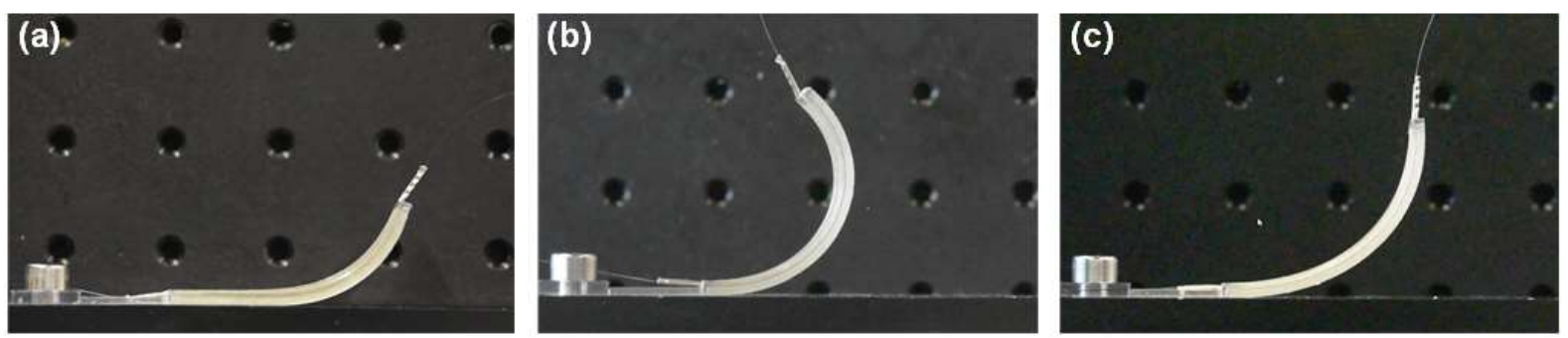

Fig. 6. Effect of the thickness of the rigid mid-layer on the maximum deformation of a $60 \mathrm{~mm}$ long bending actuator. The arrangement of soft and rigid segments is schematically shown in Fig. 3a. The matrix was printed from FLX9940, and the rigid midlayer from VeroClaer. The rigid midlayer had a thickness of a) $0.0 \mathrm{~mm}$ (no rigid layer), b) $0.3 \mathrm{~mm}$, and c) $0.5 \mathrm{~mm}$. 

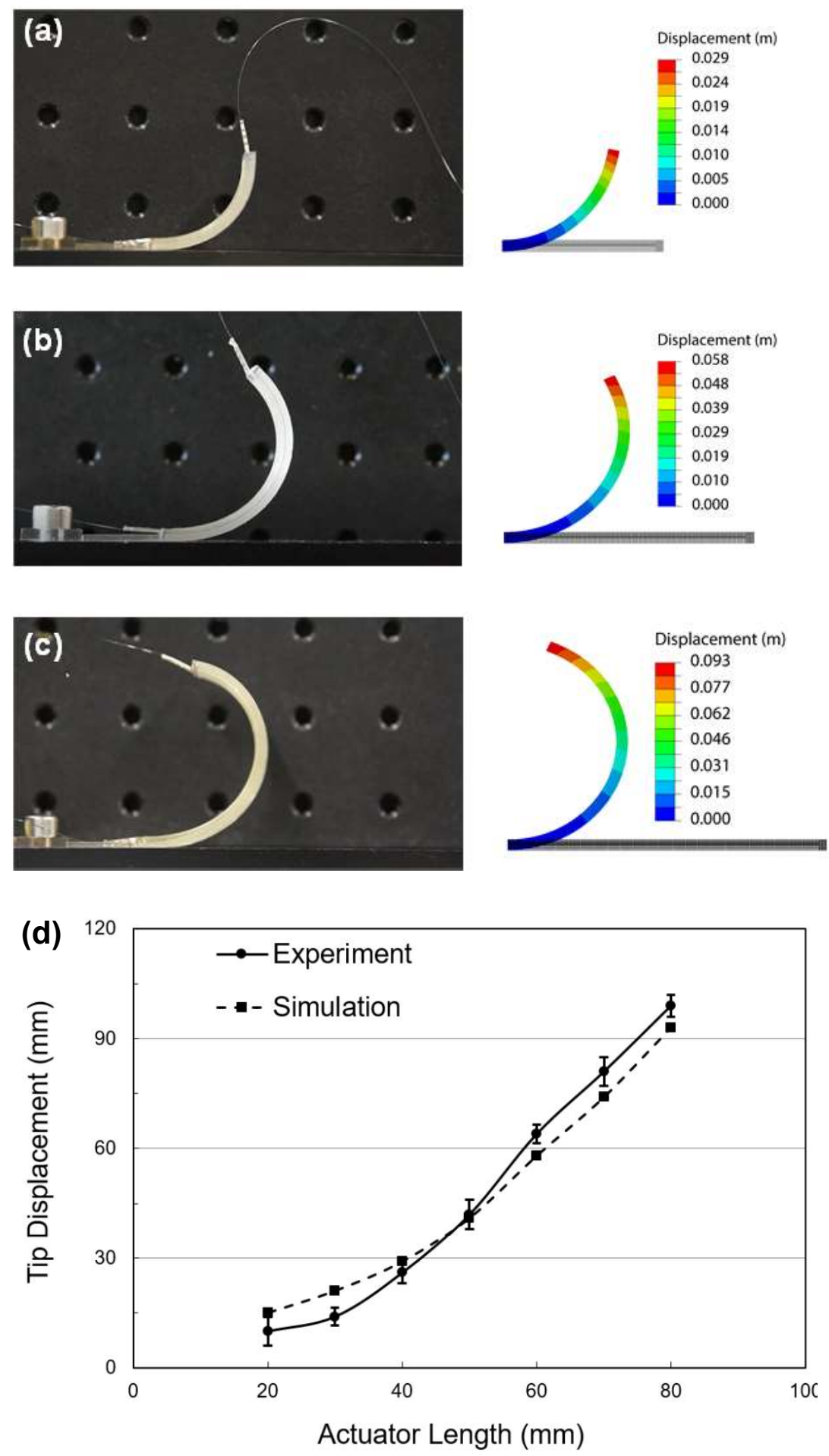

Fig. 7. Comparison of experimental and modelling results of the maximum deformation of bending actuators with lengths of a) $40 \mathrm{~mm}$, b) $60 \mathrm{~mm}$, and c) $80 \mathrm{~mm}$. The arrangement of soft and rigid segments is schematically shown in Fig. 3a. The soft matrix was printed from FLX9940, and the 0.3 $\mathrm{mm}$ thick rigid midlayer from VeroClear. d) Tip displacement versus actuator length. 

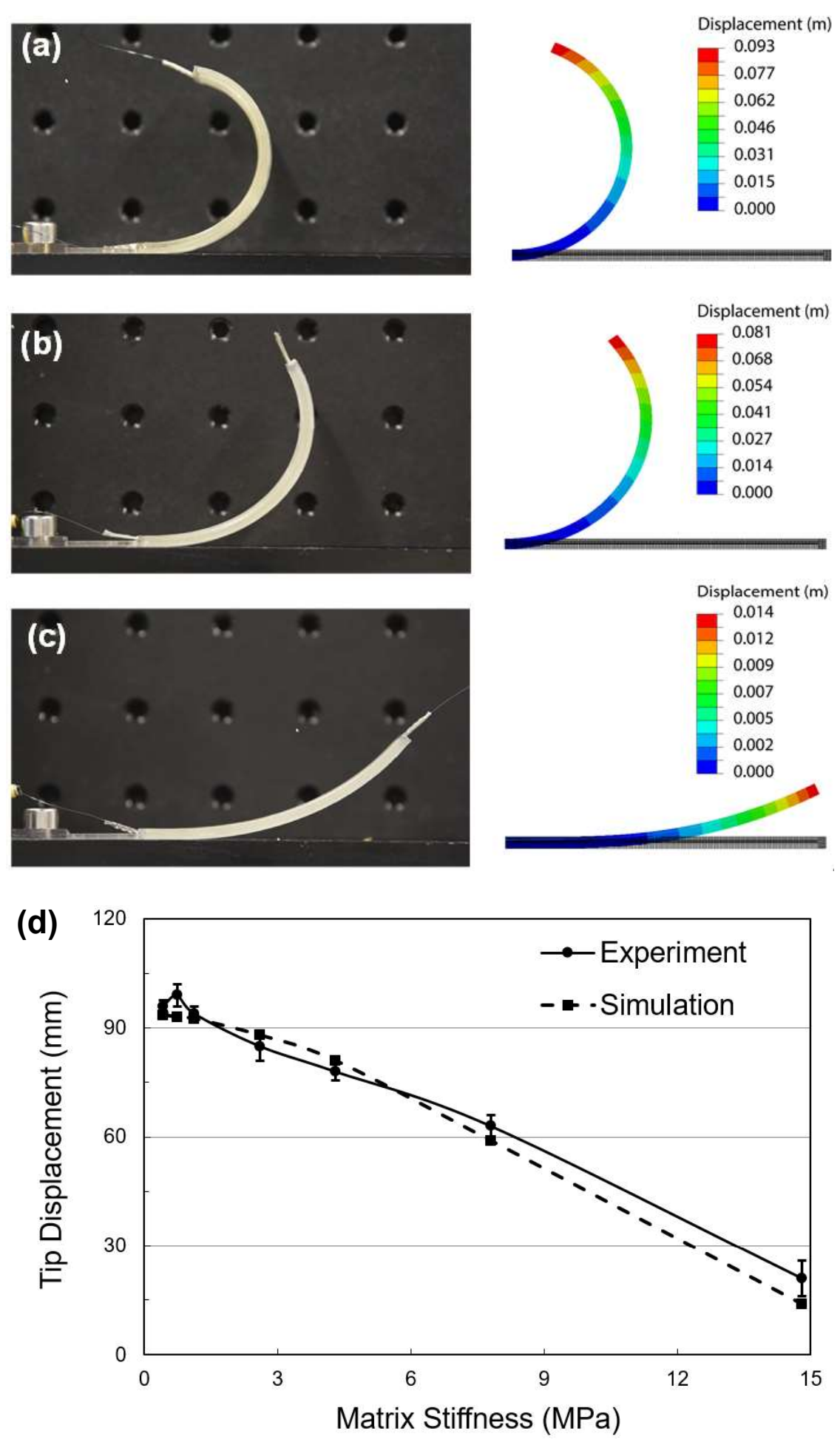

Fig. 8. Comparison of experimental and modelling results of the matrix stiffness effect on the maximum deformation of $80 \mathrm{~mm}$ long bending actuators with soft matrix printed from a) FLX9940, b) FLX9970, and c) FLX9995. The arrangement of soft and rigid segments is schematically shown in Fig. 3a. The $0.3 \mathrm{~mm}$ thick rigid midlayer was printed from VeroClear. d) Tip displacement versus the matrix stiffness. 
We also fabricated bending actuators with two hinges from the same soft digital materials, based on the design of Fig. 3b. By varying the material of the soft hinges, we can generate a variety of bending angles and stiffnesses, as measured experimentally and calculated numerically (Fig. 9). Movie S2 demonstrates the deformation of the actuator with the hinges printed from FLX9950, as in Fig. 9a. For gripper applications, higher stiffness of the hinges provides the necessary rigidity to generate effective grasping force. The values of tip displacement reported in Fig. 9d shows a good agreement between the experimental results and finite element predictions.

Multimaterial 3D printing method used in this research allows for the fabrication of the two hinges of the bending actuator from different soft digital materials to independently control the deformation of each hinge. This adds another dimension to design freedom, and enables the fabrication of finger actuators mimicking human fingers with dissimilar deformation at each hinge. Fig. 10 shows that various combinations of the hinge deformations can be obtained by using different digital soft materials for the two hinges. The inset bars show the material used to print the matrix for each section of the actuator. As observed in Fig. 10a, when the first hinge (from left) is highly stiffer than the second hinge, only the second hinge deforms. With increase of the flexibility of the first hinge, it also starts to bend. This deformation sequence can be fully reversed by printing the second hinge from a much stiffer material, as seen in Fig. 10f. 

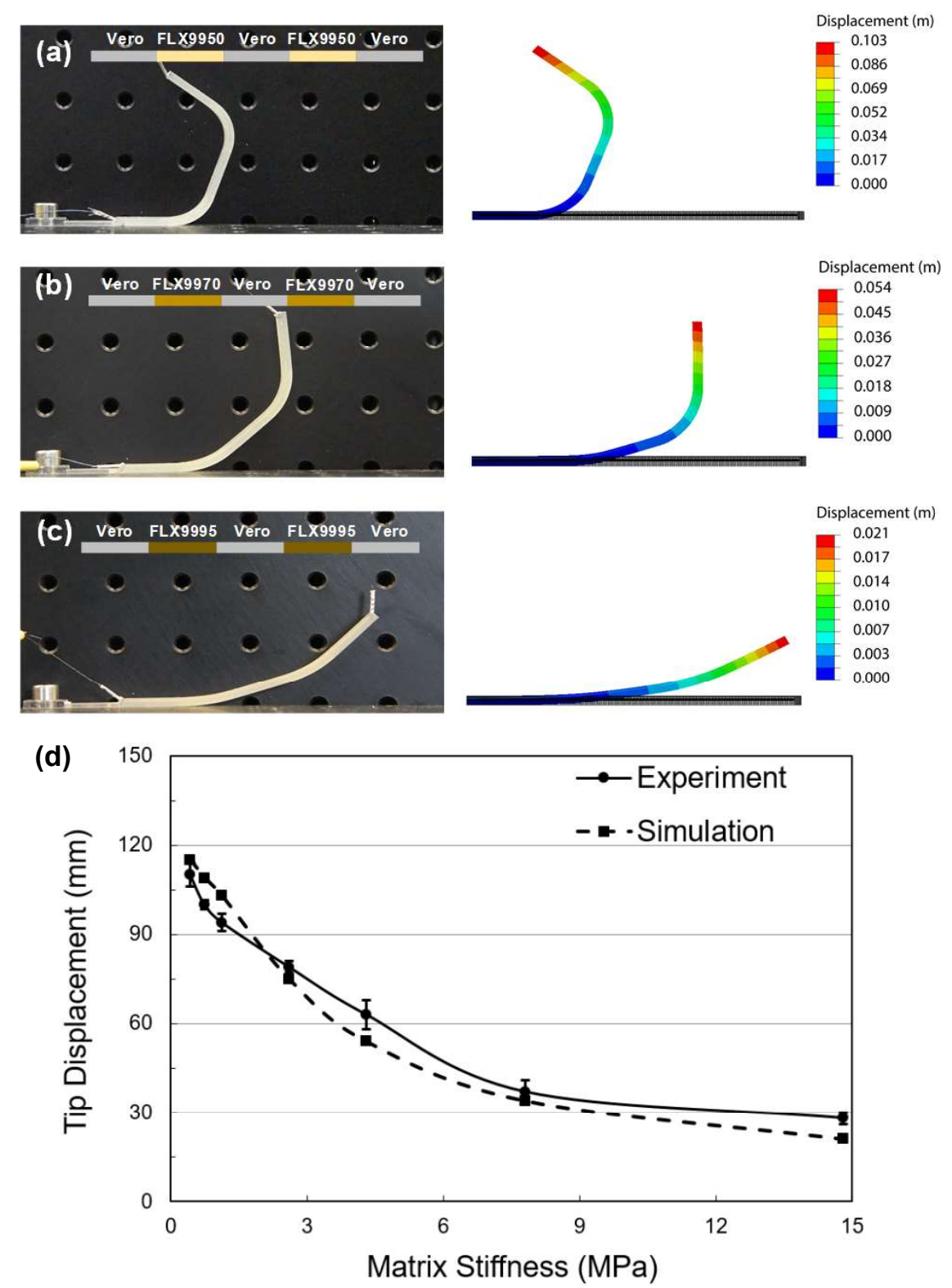

Fig. 9. Comparison of experimental and modelling results of the maximum deformation of bending actuators with two similar hinges based on the design of Fig. 3b. The total length of the actuator was $100 \mathrm{~mm}$. It consisted of 5 segments with equal lengths. The inset bar shows the material used to print the matrix for each segment. d) Tip displacement versus the matrix stiffness. 

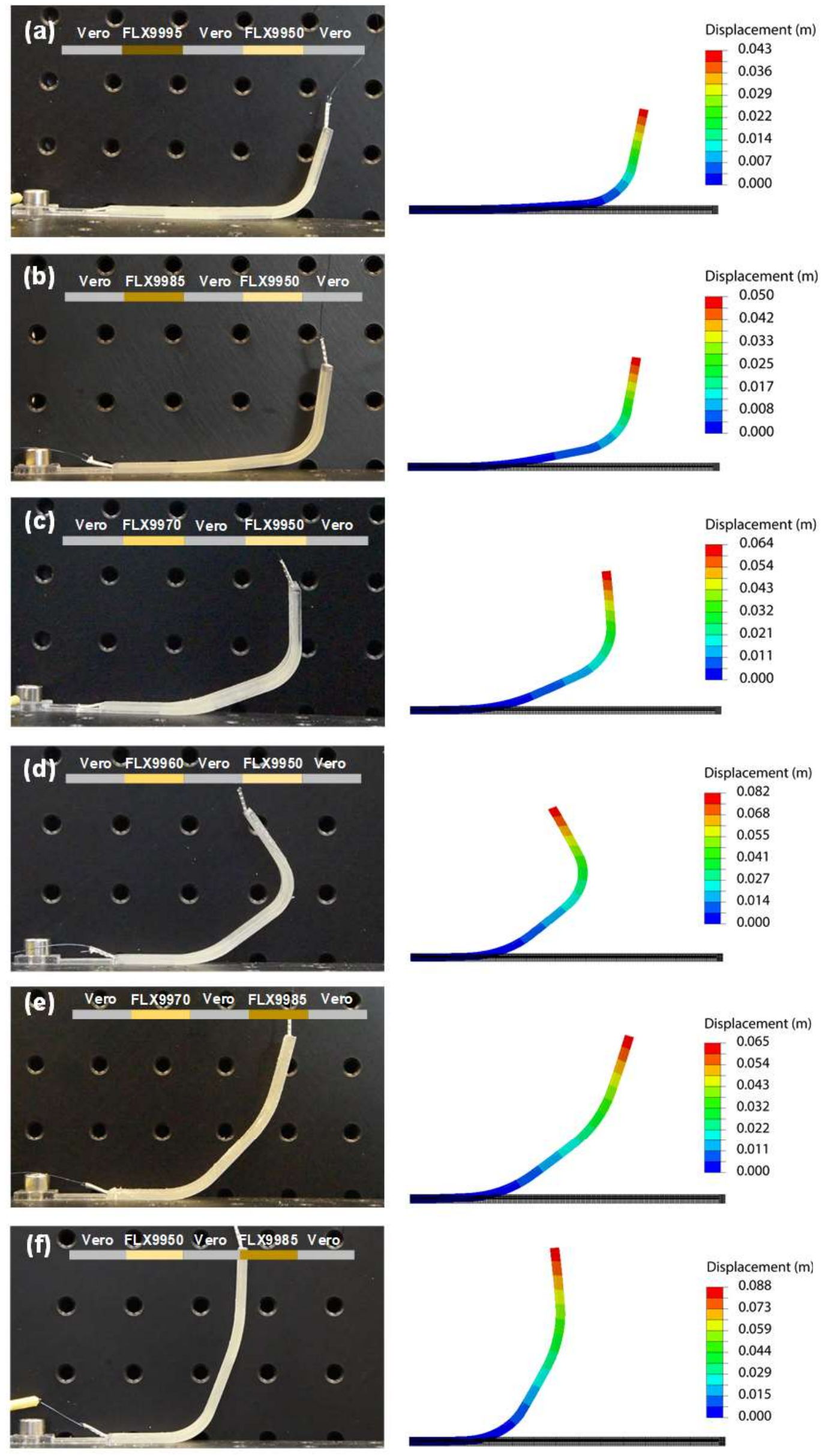
Fig. 10. Comparison of experimental and modelling results of the maximum deformation of bending actuators with two dissimilar hinges based on the design of Fig. 3b. The total length of the actuator was $100 \mathrm{~mm}$. It consisted of 5 segments with equal lengths. The inset bar shows the material used to print the matrix of each segment.

The basic designs proposed for bending deformation have many potential applications in soft robotics. To show the functionality of this approach, we designed and printed a soft gripper composed of three identical fingers. Each finger followed the design of the bending actuators of Figs 1a and 3a, with the difference that each finger had two rows of embedded SMA wires with a distance of $10 \mathrm{~mm}$ from each other. In addition, an extra rigid section is printed at the end of finger actuator to mimic the finger nail. Three identical fingers are mounted on a base, which was 3D printed from VeroClear, to create a robotic gripper. The SMA wires embedded in three figures are connected in series to ensure simultaneous actuation of all three fingers. Otherwise, each finger can be actuated with a separate SMA wire to activate each finger independently.

Fig. 11 shows a series of snapshots of effective and stable grasping of a $15 \mathrm{~g}$ cylindrical object (Movie S3). The whole grasping and releasing process was done in almost $15 \mathrm{~s}$. It is possible to generate more complex deformations by changing the soft matrix material for each hinge, or fabricating each finger with two hinges, similar to design of Fig. $3 \mathrm{~b}$ and demonstrations of Figs. 9-10, with the stiffness of each hinge independently changed by printing various digital materials with different mechanical properties.
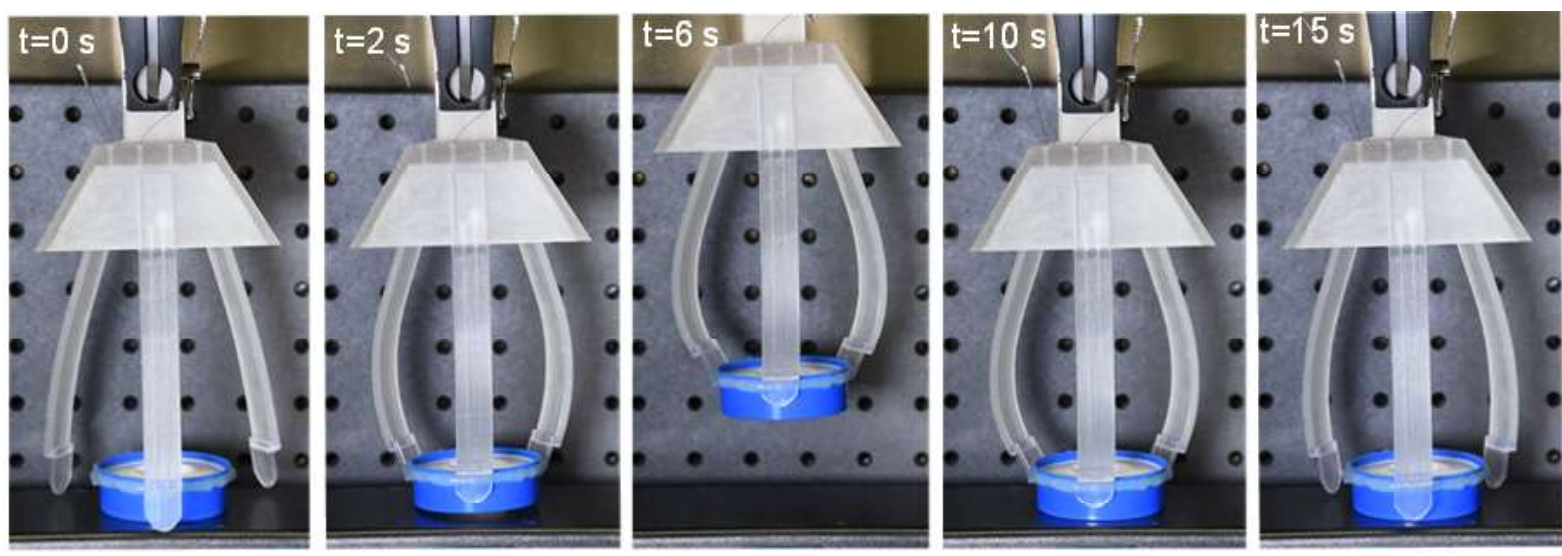

Fig. 11. Snapshots of grasping and releasing a $15 \mathrm{~g}$ cylindrical object by the soft gripper performed in $15 \mathrm{~s}$. The body of the gripper was fully printed. 


\subsection{Twisting Actuator}

One particularly useful application of multimaterial 3D printing for fabrication of SMA-based soft actuators involves creating complex 3D shapes from structures with a simple 2D original shape. As a proof-of-concept, we fabricated an actuator capable of twisting deformation by printing thin rigid fibres within the soft matrix along a diagonal direction, as specified by angle $\theta$ in Fig. 4. Rigid and soft segments of the twisting actuators were printed from VeroClear and Agilus30, respectively. Fig. 12 shows the twisting deformation of the actuator for $\theta=15^{\circ}$. More detailed deformation of this actuator is shown in Movie S4. The maximum deformation and twisting angle of the actuator can be controlled by changing $\theta$. Further experiments are ongoing to characterize this actuator, and develop a model to predict its actuation performance.
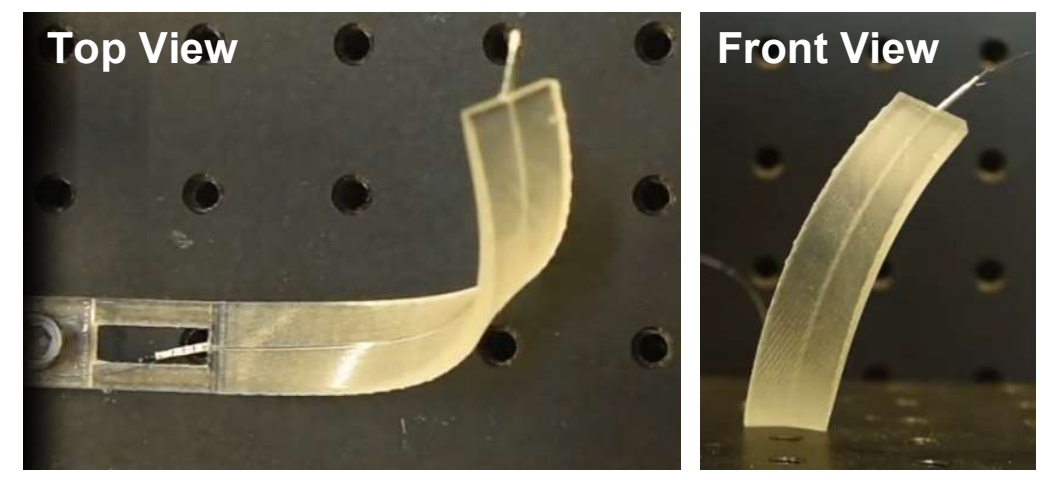

Fig. 12. Twisting actuator according to the design of the Fig. 4 . The $1 \mathrm{~mm}$ thick rigid fibers were printed from VeroClear on the actuator bottom surface at an angle $\theta=15^{\circ}$ with respect to the actuator longitudinal direction.

The main objective of the present study was to showcase the application of multimaterial 3D printing in tailoring deformation of SMA powered soft actuators by presenting several examples, and to explain different steps in fabricating and modeling. However, further studies are required to assess the actuator performance, including repeatability and actuation frequency. Also, design optimization of the printed actuators requires a rigorous mechanics-based approach to accurately describe the relationship between the properties of the anisotropic media constituting the actuator body and deformed shape of the actuator. For example, Wang et al. [38] used a minimum potential energy method 
to study the effect of material and geometric properties on twisting deformations of a cable-driven soft actuator. Incorporating nonlinear temperature dependant properties of SMA into the model proposed by Wang et al. [38] allows to investigate the SMA powered twisting actuators shown in Fig. 12 in greater detail.

\subsection{Extensional Actuator}

There is a growing demand for packable lightweight deployable structures, with an initial compact shape, that can be deployed into an extended shape under a certain external stimulus. These structures are increasingly used to deploy solar panels, antennas, and masts of satellites [9, 39-41]. However, most existing deployable structures rely on time-consuming traditional manufacturing methods, and consist of complex linkage mechanisms with numerous mechanical components, resulting in complicated and expensive assembly processes.

The third type of SMA powered actuator that we designed was a deployable structure capable of a large extensional deformation, and was designed based on the configuration introduced in Fig 5 . The design shown in Fig. 5 uses the basic design of Fig. 1b as a repeating unit with rigid segments printed on opposite sides to create two hollow pockets upon actuation. The final printed actuator shown in Fig. 13 combines two configurations of Fig. 5 placed on each other. Fig. 13 shows the initial as-fabricated shape of the actuator and its deployed configuration with five hollow pockets. The soft matrix was printed from FLX9960 with a Young's modulus of $E=2.6 \mathrm{MPa}$ (Fig. 2b), and the rigid reinforcements from VeroClear with $E=1.4 \mathrm{GPa}$ (Fig. 2d). Movie S5 shows the deployment of this actuator. The initial height of a single hollow pocket of the actuator was $6 \mathrm{~mm}$, which on average increased to $25.5 \mathrm{~mm}$ after actuation. This indicates an increase of approximately $425 \%$ in the height. Also, the width of a single pocket (in horizontal direction) changed from $75 \mathrm{~mm}$ to $65 \mathrm{~mm}$, indicating a decrease of $14 \%$ in the width. A larger number of the basic actuators can be assembled to form large-scale extensional structures. Compared with the previous deployable structures, this selfactuating structure is easy to fabricate, assemble, and deploy. 

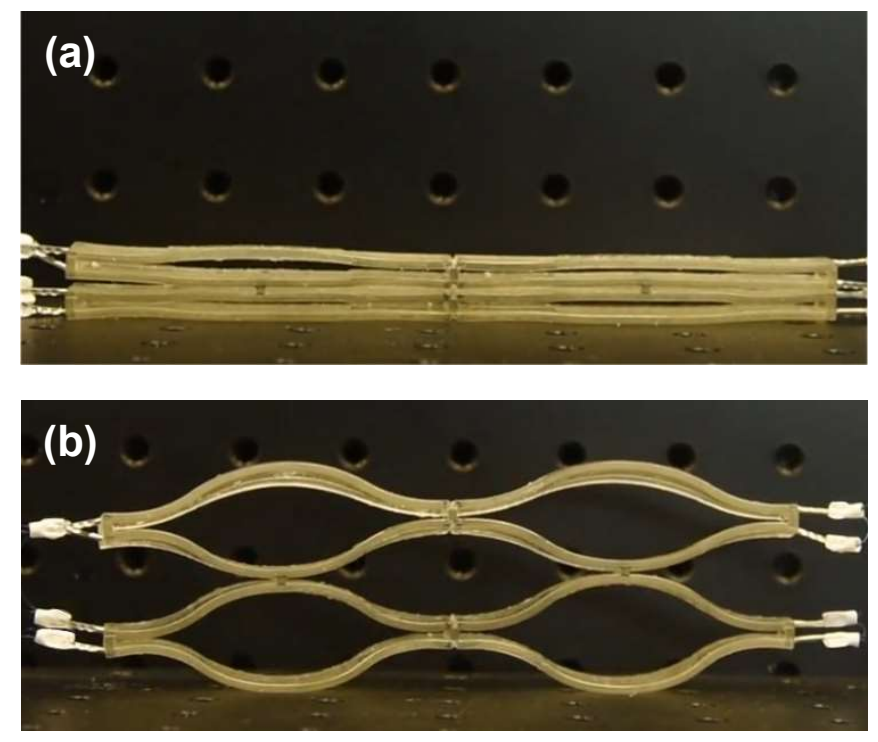

Fig. 13. Configurations of the extensional actuator following the design of Fig. 5. The soft matrix was from FLX9960, and the rigid reinforcement segments from VeroClear. a) As-fabricated compact shape of the actuator with embedded SMA wires. b) Deformed shape of the actuator.

The abovementioned demonstrations show that incorporation of SMA wires into multimaterial fully $3 \mathrm{D}$ printed parts is able to generate three-dimensional shapes. More complex shapes can be accomplished by rearrangement of rigid and soft segments to achieve desired functionality. In addition, the actuators presented here demonstrated pure bending, twisting, or extensional deformation. A combination of these deformations can be realized by utilizing the flexibility of multimaterial 3D printing to combine the basic designs shown in Figs. 3-5.

\section{Conclusions}

We introduced a design and fabrication method for soft actuators capable of tailorable large deformations. Our manufacturing method relies on a combination of materials and geometry that can be precisely controlled to achieve desired functionality. The body of the actuators was comprised of a soft matrix and a number of rigid reinforcements manufactured by multimaterial 3D printing. The one-dimensional deformation of SMA wires was then incorporated into the 3D printed soft structures to create various types of deformations. Depending on layout of the SMA wire and rigid reinforcements within the actuator, various bending, twisting and extensional deformations were demonstrated. Specifically, we used this technology to build a soft robotic gripper that represented efficient grasping and releasing performance. We also developed a finite element model which was 
able to effectively predict the actuator performance. The verified model can be used as a helpful tool to design actuators capable of complex 3D deformations, and reduce time and cost of manufacturing.

These findings prove that multimaterial 3D printing technology has a remarkable potential to advance the field of soft robotics by offering strong capacity in fabrication speed, flexibility and complexity. Unlike conventional fabrications techniques, which typically require time-consuming moulding and manual assembly processes, and are limited to simple structures, multimaterial inkjet 3D printing is able to fabricate sophisticated functional structures by depositing various combinations of photopolymers in a spatially defined manner.

\section{Acknowledgement}

The authors acknowledge support from SUTD Digital Manufacturing and Design Centre (DManD) funded by Singapore National Research Foundation (NRF). Q. G. acknowledges the support from the Agency for Science, Technology and Research (A*STAR) Public Sector Funding (PSF) (Project number 1521200086), the U.S. Office of Naval Research Global (ONRG), and the SUTD Start-up Research Grant.

\section{References}

[1] Rodrigue H, Wang W, Han M-W, Kim TJ, Ahn S-H. An Overview of Shape Memory Alloy-Coupled Actuators and Robots. Soft Robotics. 2017;4(1):3-15.

[2] Wang W, Kim N-G, Rodrigue H, Ahn S-H. Modular assembly of soft deployable structures and robots. Materials Horizons. 2017;4(3):367-76.

[3] Rodrigue H, Wang W, Kim D-R, Ahn S-H. Curved Shape Memory Alloy-based Soft Actuators and Application to Soft Gripper. Composite Structures. 2017.

[4] Wang W, Rodrigue H, Kim H-I, Han M-W, Ahn S-H. Soft composite hinge actuator and application to compliant robotic gripper. Composites Part B: Engineering. 2016;98:397-405.

[5] Wang W, Rodrigue H, Ahn S-H. Smart soft composite actuator with shape retention capability using embedded fusible alloy structures. Composites Part B: Engineering. 2015;78:507-14.

[6] Song S-H, Lee J-Y, Rodrigue H, Choi I-S, Kang YJ, Ahn S-H. $35 \mathrm{~Hz}$ shape memory alloy actuator with bending-twisting mode. Scientific reports. 2016;6.

[7] Rodrigue H, Wang W, Han M-W, Quan Y-J, Ahn S-H. Comparison of mold designs for SMA-based twisting soft actuator. Sensors and Actuators A: Physical. 2016;237:96-106.

[8] Rodrigue H, Wei W, Bhandari B, Ahn S-H. Fabrication of wrist-like SMA-based actuator by double smart soft composite casting. Smart Materials and Structures. 2015;24(12):125003.

[9] Wang W, Li C, Rodrigue H, Yuan F, Han MW, Cho M, et al. Kirigami/Origami-Based Soft Deployable Reflector for Optical Beam Steering. Advanced Functional Materials. 2017;27(7). [10] Wen L, Vogt D, Shi Z, Shen Q. Advanced Materials for Soft Robotics. Design, Fabrication, Properties and Applications of Smart and Advanced Materials. 2016:342. 
[11] Marchese AD, Katzschmann RK, Rus D. A recipe for soft fluidic elastomer robots. Soft Robotics. 2015;2(1):7-25.

[12] Rodrigue H, Wang W, Bhandari B, Han M-W, Ahn S-H. SMA-based smart soft composite structure capable of multiple modes of actuation. Composites Part B: Engineering. 2015;82:152-8. [13] Hartl D, Mabe J, Benafan O, Coda A, Conduit B, Padan R, et al. Standardization of shape memory alloy test methods toward certification of aerospace applications. Smart Materials and Structures. 2015;24(8):082001.

[14] Lecce L. Shape memory alloy engineering: for aerospace, structural and biomedical applications: Elsevier; 2014.

[15] Auricchio F, Boatti E, Conti M. SMA biomedical applications. Shape Memory Alloy Engineering: Elsevier; 2015. p. 307-41.

[16] Lagoudas DC, Rediniotis OK, Khan MM. Applications of shape memory alloys to bioengineering and biomedical technology. Scattering Theory and Biomedical Engineering Modelling and Applications: World Scientific; 2000. p. 195-207.

[17] Du Y, Liu B, Xu M, Dong E, Zhang S, Yang J. Dynamic characteristics of planar bending actuator embedded with shape memory alloy. Mechatronics. 2015;25:18-26.

[18] Smith C, Villanueva A, Joshi K, Tadesse Y, Priya S. Working principle of bio-inspired shape memory alloy composite actuators. Smart Materials and Structures. 2010;20(1):012001.

[19] Teoh J, An J, Chua C, Lv M, Krishnasamy V, Liu Y. Hierarchically self-morphing structure through 4D printing. Virtual and Physical Prototyping. 2017;12(1):61-8.

[20] Ding Z, Yuan C, Peng X, Wang T, Qi HJ, Dunn ML. Direct 4D printing via active composite materials. Science Advances. 2017;3(4):e1602890.

[21] Mao Y, Ding Z, Yuan C, Ai S, Isakov M, Wu J, et al. 3D printed reversible shape changing components with stimuli responsive materials. Scientific reports. 2016;6:24761.

[22] Bodaghi M, Damanpack A, Liao W. Self-expanding/shrinking structures by 4D printing. Smart Materials and Structures. 2016;25(10):105034.

[23] Mao Y, Yu K, Isakov MS, Wu J, Dunn ML, Qi HJ. Sequential self-folding structures by 3D printed digital shape memory polymers. Scientific reports. 2015;5:13616.

[24] Bartlett NW, Tolley MT, Overvelde JT, Weaver JC, Mosadegh B, Bertoldi K, et al. A 3D-printed, functionally graded soft robot powered by combustion. Science. 2015;349(6244):161-5.

[25] Ge Q, Dunn CK, Qi HJ, Dunn ML. Active origami by 4D printing. Smart Materials and Structures. 2014;23(9):094007.

[26] Ge Q, Qi HJ, Dunn ML. Active materials by four-dimension printing. Applied Physics Letters. 2013;103(13):131901.

[27] Chen T, Mueller J, Shea K. Integrated design and simulation of tunable, multi-state structures fabricated monolithically with multi-material 3D printing. Scientific reports. 2017;7:45671.

[28] Stanković T, Mueller J, Egan P, Shea K. A generalized optimality criteria method for optimization of additively manufactured multimaterial lattice structures. Journal of Mechanical Design.

2015;137(11):111405.

[29] Wagner M, Chen T, Shea K. Large Shape Transforming 4D Auxetic Structures. 3D Printing and Additive Manufacturing. 2017;4(3):133-42.

[30] Umedachi T, Vikas V, Trimmer B. Softworms: the design and control of non-pneumatic, 3Dprinted, deformable robots. Bioinspiration \& biomimetics. 2016;11(2):025001.

[31] Gul JZ, Yang B-S, Yang YJ, Chang DE, Choi KH. In situ UV curable 3D printing of multi-material trilegged soft bot with spider mimicked multi-step forward dynamic gait. Smart Materials and Structures. 2016;25(11):115009.

[32] Meisel NA, Elliott AM, Williams CB. A procedure for creating actuated joints via embedding shape memory alloys in PolyJet 3D printing. Journal of Intelligent Material Systems and Structures. 2015;26(12):1498-512. 
[33] Ahn S-H, Lee K-T, Kim H-J, Wu R, Kim J-S, Song S-H. Smart soft composite: An integrated 3D soft morphing structure using bend-twist coupling of anisotropic materials. International Journal of Precision Engineering and Manufacturing. 2012;13(4):631-4.

[34] Lagoudas DC, Bo Z, Qidwai MA. A unified thermodynamic constitutive model for SMA and finite element analysis of active metal matrix composites. Mechanics of Composite Materials and Structures. 1996;3(2):153-79.

[35] Sakhaei AH, Thamburaja P. A finite-deformation-based constitutive model for high-temperature shape-memory alloys. Mechanics of Materials. 2017;109:114-34.

[36] Yap HK, Ng HY, Yeow C-H. High-force soft printable pneumatics for soft robotic applications. Soft Robotics. 2016;3(3):144-58.

[37] Han M-W, Rodrigue H, Kim H-I, Song S-H, Ahn S-H. Shape memory alloy/glass fiber woven composite for soft morphing winglets of unmanned aerial vehicles. Composite Structures.

2016;140:202-12.

[38] Wang D, Li L, Serjouei A, Dong L, Weeger O, Gu G, et al. Controllable helical deformations on printed anisotropic composite soft actuators. Applied Physics Letters. 2018;112(18):181905.

[39] Felton S, Tolley M, Demaine E, Rus D, Wood R. A method for building self-folding machines. Science. 2014;345(6197):644-6.

[40] Huang H, Deng Z, Li B. Mobile assemblies of large deployable mechanisms. Journal of Space Engineering. 2012;5(1):1-14.

[41] Zhang R, Guo X, Liu Y, Leng J. Theoretical analysis and experiments of a space deployable truss structure. Composite Structures. 2014;112:226-30. 
Saeed Akbari is a research engineer at RISE Research Institutes of Sweden. He received his $\mathrm{PhD}$ from University of Toronto, Canada, at 2017. His research interests include additive manufacturing, active materials, polymer composites $\&$ nanocomposites, electronic packaging and board level reliability tests.

Amir Hosein Sakhaei is a postdoctoral research fellow at University of Exeter, UK. He obtained his $\mathrm{PhD}$ from National University of Singapore at 2014. His research focuses on theoretical and computational mechanics of advanced materials, continuum mechanics, finite element simulations, and product design using advanced manufacturing.

Sahil Panjwani is a Research Assistant at Singapore University of Technology and Design (SUTD). His research interests incluse robotics, Computer-Aided-Engineering (CAE), and 3D printing. He received his Bachelor of Technology from Manipal University Jaipur, India, at 2017.

Kavin Kowsari is a postdoctoral research fellow at Singapore University of Technology and Design (SUTD). He received his PhD from University of Toronto, Canada, at 2017. His research areas are additive manufacturing, 3D/4D printing, biofabrication, soft robotics, and computational fluid dynamics.

Ahmad Serjouei is a senior researcher in additive manufacturing at Institute for Innovation in Sustainable Engineering, Derby, UK. He received his PhD from Nanyang Technological University, Singapore, at 2014. His research interests rely on additive manufacturing, 3D and 4D printing of polymers, and modeling of solid structures.

Qi Ge is an Assistant Professor Singapore University of Technology and Design (SUTD). He received his $\mathrm{PhD}$ from University of Colorado Boulder at 2012. He is a well-known research in 4D printing, shape memory polymers, and functional printable materials. 